\title{
Agomelatine Drug Utilisation Study in Selected European Countries: A Multinational, Observational Study to Assess Effectiveness of Risk-Minimisation Measures
}

\author{
Emmanuelle Jacquot ${ }^{1} \cdot$ Estelle Collin $^{1} \cdot$ Amy Ladner $^{2} \cdot$ Anita Tormos $^{3} \cdot$ Lynne Hamm $^{2} \cdot$ Susana Perez-Gutthann ${ }^{3}$. \\ Lia Gutierrez ${ }^{3} \cdot$ Costel Chirila $^{2} \cdot$ Nicolas Deltour $^{1}$
}

Published online: 11 July 2019

(c) The Author(s) 2019

\begin{abstract}
Background Hepatotoxic reactions are an important identified risk listed in the agomelatine risk management plan. This postauthorisation safety study evaluated the effectiveness of additional risk-minimisation measures (aRMMs) for agomelatine. Objective The objective of this study was to evaluate, among physicians prescribing agomelatine and their patients, liver function monitoring adherence, compliance with contraindications and patients' reasons for non-compliance with liver monitoring.

Methods A non-interventional cohort study was conducted among adults initiating agomelatine in routine clinical practice in Denmark, France, Germany and Spain through a retrospective medical record abstraction (MRA) before and after implementation of aRMMs and a cross-sectional patient survey.

Results Fifty-four sites contributed data on 437 and 404 patients in the before- and after-RMM periods, and 237 patients completed the survey. No patient had cirrhosis in either study period; $98.2 \%$ of patients in the before- and $98.0 \%$ in the afterRMM period had no active liver disease reported at initiation or during treatment. Compliance to contraindicated medications was $>99 \%$ in both periods. The adherence to the liver-monitoring regimen was similar in both periods $(15.1 \%$ before RMM and $16.3 \%$ after RMM). In the after-RMM period, $25.2 \%$ of patients had a liver test before or at treatment initiation; $61.5 \%$ had a liver test during treatment. Among patients surveyed who did not have a blood test before treatment initiation or during treatment, the most frequently cited reason was a test ordered but not yet performed.

Conclusions The overall adherence to liver-monitoring recommendations remained weakly influenced by aRMMs. However, patients treated with agomelatine are in compliance with relevant contraindications.
\end{abstract}

\section{Introduction}

Agomelatine $\left(\right.$ Valdoxan ${ }^{\circledR} /$ Thymanax $^{\circledR}$ ) is a melatonergic agonist (MT1 and MT2 receptors) and a serotonin 5- $\mathrm{HT}_{2 \mathrm{C}}$ receptor antagonist indicated for major depressive episodes in adults. Agomelatine is covered by a European

Electronic supplementary material The online version of this article (https://doi.org/10.1007/s40290-019-00291-2) contains supplementary material, which is available to authorized users.

Lynne Hamm

lhamm@rti.org

Institut de Recherches Internationales Servier, Paris, France

2 RTI Health Solutions, 200 Park Offices Drive, Research Triangle Park, Durham, NC 27709, USA

3 RTI Health Solutions, Barcelona, Spain

\section{Key Points}

A post-authorisation safety study was conducted to evaluate the effectiveness of additional risk-minimisation measures (aRMMs) among physicians prescribing agomelatine and their patients with respect to livermonitoring adherence, compliance with contraindications and patients' reasons for non-compliance with liver monitoring.

Although adherence to liver-monitoring recommendations remained weakly influenced by aRMMs, patients treated with agomelatine are in compliance with relevant contraindications. 
risk management plan identifying hepatotoxic reactions as an important identified risk. In randomised clinical trials (RCTs) of $\geq 9500$ patients, $1.3 \%$ of patients treated with agomelatine $25 \mathrm{mg}$ and $2.5 \%$ of patients treated with agomelatine $50 \mathrm{mg}$ showed elevated transaminases $>3$ times the upper limit of normal (ULN), compared with $0.5 \%$ with placebo. Hepatic reactions in RCTs consisted mainly of asymptomatic reversible transaminase elevations, observed within the first months of treatment with no case of severe hepatic dysfunction [1]. In the initial marketing authorisation (MA) for agomelatine in 2009, risk-minimisation measures (RMMs), including contraindications in patients with hepatic impairment (i.e. cirrhosis or active liver disease), precautions of use, liver testing at initiation and at 6,12 and 24 weeks after initiation, and a physician's guide to prescribing, were requested to minimise the risk of hepatotoxicity. Since MA, spontaneous cases of hepatic events, including cases of severe hepatic dysfunctions, have been reported. The RMMs were expanded in 2012 and 2013 to include liver testing 3 weeks after treatment initiation and in the event of dosage increase, and contraindication in patients with transaminases $>3 \times U L N$.

Updated physicians' guides were distributed in Europe, and Dear Health Care Provider (DHCP) letters were sent in 2012 and 2013. In 2014, suboptimal adherence to liver monitoring at initiation and during treatment were observed in a prospective observational cohort study. Based on these results, together with updated post-marketing data, the European Medicines Agency (EMA) Pharmacovigilance Risk Assessment Committee (PRAC) requested additional RMMs (aRMMs). The aRMMs specified that liver testing required at treatment initiation had to be performed before the start of agomelatine treatment, such that liver function would be monitored before treatment and at 3, 6, 12 and 24 weeks. The message regarding precautions of use in patients with risk factors for hepatic injury or contraindicated co-medications (fluvoxamine and ciprofloxacin) was also reinforced [2]. The prescriber guide was then distributed twice a year, and a patient booklet was introduced and distributed to patients by their prescriber. The booklet was intended to inform patients of the potential for liver toxicity and of liver-monitoring requirements, with the aim of promoting patients' active role in adherence to the monitoring programme.

This post-authorisation safety study (PASS) was required [2] to assess the effectiveness of the aRMMs in terms of adherence to liver monitoring and compliance with relevant contraindications (via a medical record abstraction [MRA]) and evaluate reasons for non-compliance with liver function monitoring from the patient's perspective (via a survey).

\section{Methods}

\subsection{Study Design and Setting}

This non-interventional cohort study collected data from adult patients initiating agomelatine treatment in routine clinical practice in Denmark, France, Germany and Spain. The selection of countries was driven by the sales volume of agomelatine in Europe, the distribution of prescriptions according to specialties and practice settings, and the desire for a diverse geographic representation of European countries.

Figure 1 presents an overview of the study scheme. The prescriber's guide and the patient booklet were disseminated in countries participating in this study from December 2014 through July 2015. The study comprised two independent components: a retrospective MRA and a cross-sectional patient survey. The MRA included two periods. The "beforeRMM' period collected information from January 2013 to November 2014, corresponding to a time period before adoption of the latest aRMMs. The 'after-RMM' period (February-August 2015 to November 2016) captured information beginning 1-2 months after the latest implementation of the new aRMMs through to the month before study activities were initiated. Recruitment of patients for the survey was performed in a cross-sectional manner.

Physicians were sampled from lists of more than 19,000 general practitioners (GPs) and psychiatrists. The distribution of the targeted physicians aimed to reflect prescribing patterns in each country, with 60 active centres planned overall. Eligible physicians had at least five patients per study period for GPs and eight patients for specialists. To achieve a balanced representation across settings and specialties and to avoid cluster effects, the number of patients per centre and period was limited to ten for GPs and 15 for specialists. Centres began recruiting patients once all appropriate approvals were received.

\subsection{Study Population}

Eligible patients were aged $\geq 18$ years at treatment initiation and were not participating in a clinical trial of agomelatine.

For the MRA, eligible patients had initiated agomelatine during at least one of the study periods and provided written informed consent (except in France, where not objecting to the processing of the personal data was acceptable for the MRA). Patients with documented use of agomelatine in the 6 months before the first recorded prescription in a study period were ineligible. All eligible patients were identified and received information on the study, and sites were instructed to collect the data of the first ten or 15 patients 
Fig. 1 Study overview. $D H P C$ Dear Health Care Professional, $E U$ European Union, $R M M$ riskminimisation measure, SmPC Summary of Product Characteristics

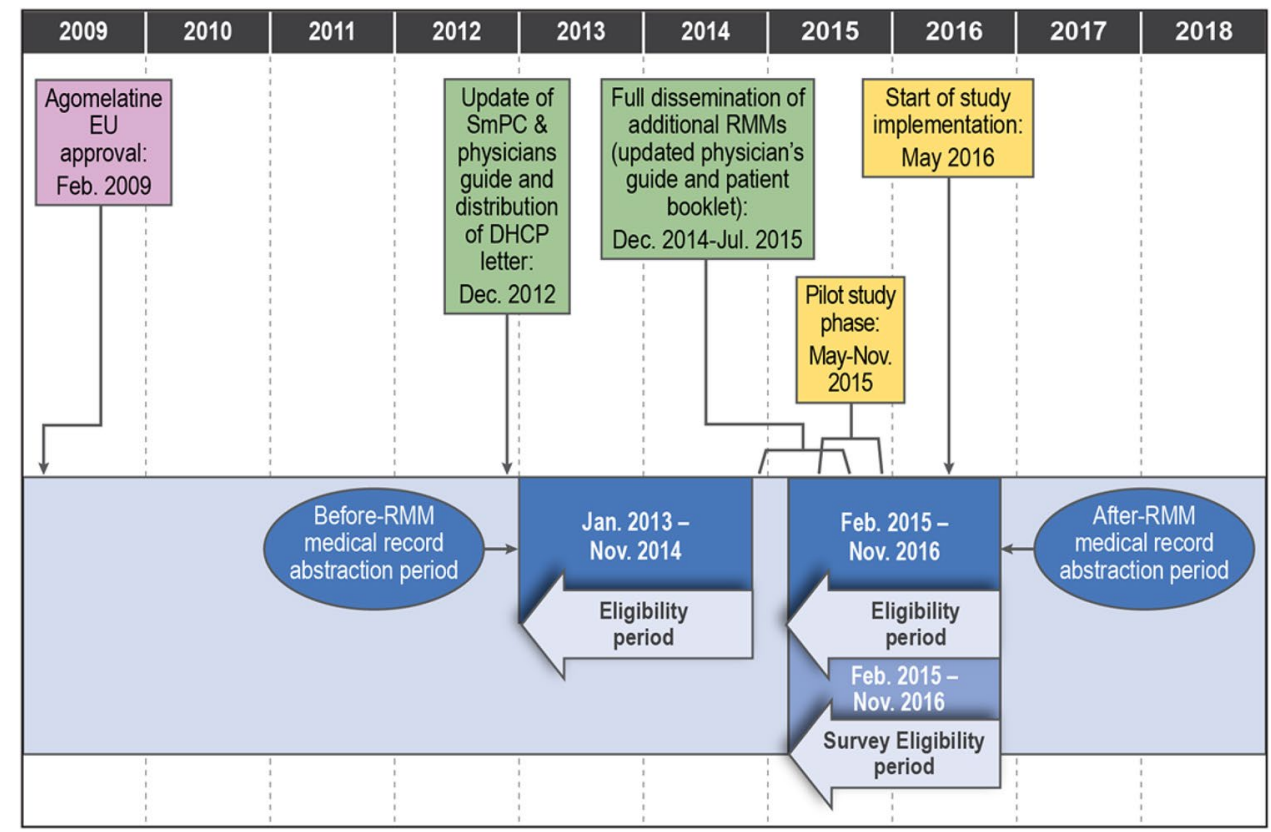

who returned their signed informed consent. Overall, 600 patients were initially targeted for each period. However, according to the calculated precision estimates, a study size of 400 patients per study period was considered acceptable to meet the study objective (Electronic Supplementary Material, Figure S-1), with an absolute precision of $6 \%$ for the worst precision scenario of 50\% adherence to liver testing recommendations, yielding a $95 \%$ confidence interval (CI) of 44-56.

For the survey, patients were recruited and completed the questionnaire during a routine visit. Eligible patients had initiated treatment with agomelatine after the dissemination of the patient booklet in each country (corresponding to the after-RMM period), were currently using or had discontinued agomelatine no more than 3 months before the survey date, and provided written informed consent. Overall, 400 patients were targeted, but a study size of 300 was considered acceptable to meet the survey objectives.

\subsection{Study Variables}

For the MRA, data were abstracted on patients' characteristics, including co-morbidities and medications, agomelatine prescription, and timing and results of liver function tests.

For the patient survey, data were collected on patients' characteristics, agomelatine treatment, receipt of the patient booklet, knowledge of the key liver safety information, performance of liver testing and reasons for non-compliance (if applicable).

\subsection{Statistical Analyses}

All statistical analyses were performed using $\mathrm{SAS}^{\circledR}$ version 9.4 (SAS Institute, Cary, NC, USA).

Frequency distributions for categorical variables and summary statistics for continuous variables were calculated. Analyses were conducted by country and overall.

Data on agomelatine treatment duration were derived from prescription dates and drug supply information. Considering the observational study design and the possibility for missing data, two treatment duration definitions were used: theoretical and empirical. The theoretical treatment duration - the more conservative approach-was calculated from the first prescription start date to the earliest date among end date of the last prescription, discontinuation date or end of the concerned study period. For the dose-escalation analysis, the theoretical treatment duration before dose escalation was derived from the start date of the first prescription to the start date of the first dose-escalation prescription. After dose escalation, the theoretical treatment duration was calculated from the start date of the dose escalation to the earliest date among end of the last prescription, discontinuation date or end of study period.

The empirical duration was similarly defined but accounted for treatment overlap and treatment stopping. Specifically, for any two consecutive prescriptions that overlapped, the later prescription was shifted such that its start date was the day after the end date of the previous prescription, and the overlapping days of supply were added 
at the end. If a gap $>30$ days occurred between any two consecutive prescriptions, then the first of these two prescriptions was the last to be considered (i.e. all later prescriptions, including dose escalations, were discarded). If a gap of $\leq 30$ days occurred between any two prescriptions, then treatment was considered uninterrupted. Because the results using theoretical and empirical duration definitions were similar due to the small number of patients with prescription gaps $>30$ days, only the results using the theoretical duration are presented.

The primary analysis evaluated adherence to one pretreatment test plus at least one test during treatment, considered the minimum number of tests necessary to evaluate potential liver toxicity. For this analysis, adherence was defined as the proportion of patients with at least one liver test performed between 4 weeks before and 3 days after treatment initiation (M1 criterion) and at least one test performed during treatment from 2 to 28 weeks after treatment initiation (M2 criterion). The secondary analysis performed a more focused evaluation on full adherence to the actual liver monitoring required. In the secondary analysis, adherence was defined with a stricter composite criterion, combining the test before initiation ( $\mathrm{S} 1$ criterion, using a time window identical to the $\mathrm{M} 1$ criterion) but also requiring the performance of all tests required during treatment ( $\mathrm{S} 2$ criterion; detailed time windows are provided in Table 1) in patients without or until dose escalation. In patients with dose escalation, adherence was studied using a composite criterion combining at least one test before dose escalation (E1 criterion) and one test after dose escalation (E2 criterion). Prevalence of adherence and $95 \%$ CIs were estimated for both treatment duration definitions. The difference of the estimated prevalence of adherence between both periods and its $95 \%$ CI was calculated. The $95 \%$ CI for the difference was calculated using the Wilson score method for difference in proportion [3]. For primary and secondary analyses, a Pearson's Chi-square or Fisher's exact test was used to test the differences in proportions between the two study periods [4].
Table 1 Prevalence of adherence to the livermonitoring test regimen based on the theoretical treatment duration definition by study period: overall patients

\begin{tabular}{|c|c|c|c|}
\hline \multirow{2}{*}{$\begin{array}{l}\text { Theoretical treatment duration } \\
\text { Main analysis }\end{array}$} & \multicolumn{3}{|c|}{ Patients who met liver-monitoring test adherence criterion [\% (95\% CI)] } \\
\hline & M1 $^{\mathrm{a}}$ & $\mathbf{M} 2^{\mathrm{b}}$ & Composite criterion: M1 and M2 \\
\hline \multicolumn{4}{|l|}{ Overall } \\
\hline Before RMM $(n=378)$ & $24.1(20.0-28.6)$ & $56.3(51.3-61.3)$ & $15.1(11.8-19.0)^{\mathrm{c}}$ \\
\hline After RMM $(n=361)$ & $25.2(21.0-29.9)$ & $61.5(56.4-66.4)$ & $16.3(12.9-20.5)^{\mathrm{c}}$ \\
\hline \multicolumn{4}{|l|}{ Secondary analyses } \\
\hline Stricter definition & S1 & $\mathbf{S 2}^{\mathrm{d}}$ & Composite criterion: S1 and S2 \\
\hline \multicolumn{4}{|l|}{ Overall } \\
\hline Before RMM $(n=378)$ & $24.1(20.0-28.6)$ & $19.0(15.4-23.3)$ & $7.4(5.2-10.5)^{\mathrm{e}}$ \\
\hline After RMM $(n=361)$ & $25.2(21.0-29.9)$ & $21.6(17.7-26.1)$ & $7.2(5.0-10.3)^{\mathrm{e}}$ \\
\hline Dose escalation & $\mathbf{E 1}^{\mathrm{f}}$ & $\mathbf{E} 2^{\mathrm{g}}$ & Composite criterion: $\mathrm{E} 1$ and $\mathrm{E} 2$ \\
\hline \multicolumn{4}{|l|}{ Overall } \\
\hline Before RMM $(n=58)$ & $31.0(20.6-43.8)$ & $50.0(37.5-62.5)$ & $20.7(12.3-32.8)^{\mathrm{h}}$ \\
\hline After RMM $(n=58)$ & $44.8(32.7-57.5)$ & $67.2(54.4-77.9)$ & $39.7(28.1-52.5)^{\mathrm{h}}$ \\
\hline
\end{tabular}

$R M M$ risk-minimisation measure

${ }^{a}$ At least one liver test performed between 4 weeks before and 3 days after the initiation of agomelatine treatment

${ }^{\mathrm{b}}$ At least one liver test performed between 2 and 28 weeks after treatment initiation and while on treatment ${ }^{c}$ Difference chi-square test ( $\left.95 \% \mathrm{CI}\right)-0.013$ ( -0.07 to 0.04$), p=0.64$

${ }^{\mathrm{d}}$ All required tests performed according to the treatment duration and the timings as defined in the summary of product characteristics: liver test 3 weeks after treatment initiation within the time window (week 2-week 4) after treatment start; liver test 6 weeks after treatment initiation within the time window (week 5-week 8) after treatment start; liver test 12 weeks after treatment initiation within the time window (week 9-week 16) after treatment start; and liver test 24 weeks after treatment initiation within the time window (week 17-week 28) after treatment start

${ }^{\mathrm{e}}$ Difference chi-square test (95\% CI): -0.002 ( -0.04 to 0.04 ), $p=0.92$

${ }^{\mathrm{f}}$ At least one liver test before or at dose escalation, defined as within 1 week before or 1 week after dose escalation

${ }^{\mathrm{g}}$ At least one liver test after dose escalation, defined as 2-28 weeks after dose escalation and during treatment

${ }^{\mathrm{h}}$ Difference chi-square test (95\% CI): $-0.190(-0.34$ to -0.02$), p=0.03$ 
Planned sensitivity analyses assessed the impact of the shared care management (when a patient was not managed only by the enrolling physician) on missing data and the prevalence of adherence to the liver monitoring.

\section{Results}

A total of 19,301 physicians were invited by telephone, mail or e-mail to participate in the study. Of these, $91.4 \%$ were non-responsive and $7.6 \%$ refused to participate. Of the 191 physicians (1.0\% of those contacted) who initially expressed interest in participating, 63 were contracted and trained for the study. Of these, 54 physicians became active sites (21 GPs and 33 specialists) and recruited 437 patients in the before-RMM period and 404 patients in the after-RMM period of the MRA (35 patients contributed data to both periods). Owing to recruitment challenges and the aim to meet the regulatory agreed timelines for study completion, the target samples of 600 patients by period were not reached, but the study size was considered sufficient to meet the study objective. Females represented $65.7 \%$ of patients in the before-RMM and $67.3 \%$ in the after-RMM period. The largest proportion of patients was aged $46-65$ years $(49.4 \%$ and $45.0 \%$ in the before- and after-RMM period, respectively). Patient characteristics were similar between periods (Electronic Supplementary Material, Table S-1). Shared care was reported for $19.7 \%$ of patients in the before-RMM period and $23.8 \%$ in the after-RMM period. According to theoretical treatment duration, $66.6 \%$ of patients in the before-RMM period and $72.5 \%$ in the after-RMM period had at least one liver function test performed by a physician at the recruiting practice, and $21.7 \%$ in the before-RMM period and $26.0 \%$ in the after-RMM period had at least one liver function test performed by a physician outside the recruiting practice. Further, $9.6 \%$ of patients in the before-RMM period and $10.4 \%$ in the after-RMM period had liver tests ordered but not performed. Overall, $72.5 \%$ of patients in the beforeRMM period and $79.5 \%$ in the after-RMM period had liver function test results that had complete dates for all reported tests.

For the survey, 254 patients were approached; 237 (93.3\%) completed the survey, of whom $62.9 \%$ were female. The largest proportion of patients $(47.3 \%)$ was in the group aged 46-65 years.

\subsection{Compliance with Contraindications and Precautions of Use}

Based on data recorded in the medical records, no patient in either the before-RMM or after-RMM period had records of cirrhosis at initiation or during agomelatine treatment. Overall, $98 \%$ of patients in each period were free of any chronic or acute liver disease at initiation or during agomelatine treatment (Table 2). Compliance with contraindicated medications (fluvoxamine and ciprofloxacin) was above $99 \%$ in both study periods (Table 2). No patients with a liver test before or at initiation had a transaminase result $>3 \times$ ULN.

Regarding co-morbidities identified as risk factors for hepatic injury and precautions of use, $99 \%$ of patients in either period had no records of non-alcoholic fatty liver disease and approximately $95 \%$ had no alcohol use disorder and no diabetes mellitus (either type 1 or type 2). Approximately $81 \%$ were not reported by physicians as either obese or overweight (Table 3); however, the proportion of patients with a calculated body mass index $(\mathrm{BMI}) \geq 25$, corresponding to overweight or obesity, was $36.8 \%$ in the before-RMM period and $36.4 \%$ in the after-RMM period (BMI was missing for approximately $30 \%$ of patients in either study period).

\subsection{Adherence to Liver-Monitoring Recommendations}

In the primary analysis, based on theoretical treatment duration, the percentage of patients with at least one test before or at initiation and during agomelatine treatment was similar in both study periods (before-RMM period: $15.1 \%$ [95\% CI 11.8-19.0]; after-RMM period: $16.3 \%$ [95\% CI 12.9-20.5]) (Table 1). Specifically, 24.1\% (95\% CI 20.0-28.6) of patients in the before-RMM period and $25.2 \%$ (95\% CI 21.0-29.9) in the after-RMM period had a liver test before or at treatment initiation (M1 criterion). During treatment, 56.3\% (95\% CI 51.3-61.3) of patients in the before-RMM period and $61.5 \%$ (95\% CI 56.4-66.4) in the after-RMM period had at least one liver test. Calculations based on empirical treatment duration yielded similar results (data not shown).

In the secondary analysis applying more stringent criteria, the percentage of patients who had required liver tests according to the liver-monitoring schedule was also similar in both periods (before-RMM period: $7.4 \%[95 \%$ CI 5.2-10.5]; after-RMM period: 7.2\% [95\% CI 5.0-10.3]). Compliance to testing before treatment ( $\mathrm{S} 1$ criterion) was identical to the M1 criterion. With the S2 criterion, each test required during treatment was performed in approximately $30 \%$ of patients in either study period (Electronic Supplementary Material, Table S-2). Among patients with dose escalation, adherence to liver-monitoring recommendations increased between study periods from $20.7 \%(95 \%$ CI 12.3-32.8) to reach $39.7 \%$ (95\% CI 28.1-52.5) in the after-RMM period. 
Table 2 Prevalence of contraindications by study period: overall patients

\begin{tabular}{|c|c|c|}
\hline \multirow{2}{*}{$\begin{array}{l}\text { Medical conditions or medications present at initial agomelatine } \\
\text { prescription and/or that occurred while taking agomelatine }\end{array}$} & \multicolumn{2}{|l|}{ Overall patients $[n(\%)]$} \\
\hline & $\begin{array}{l}\text { Before-RMM period } \\
(n=437)\end{array}$ & $\begin{array}{l}\text { After-RMM } \\
\text { period }(n=404)\end{array}$ \\
\hline \multicolumn{3}{|l|}{ Contraindicated co-morbidities } \\
\hline \multicolumn{3}{|l|}{ Active liver disease category (either acute or chronic) } \\
\hline No medical condition & $429(98.2)$ & $396(98.0)$ \\
\hline Confirmed at initiation or during treatment & $4(0.9)$ & $4(1.0)$ \\
\hline Unconfirmed $^{\mathrm{a}}$ at initiation of or during treatment & $4(0.9)$ & $4(1.0)$ \\
\hline \multicolumn{3}{|l|}{ Cirrhosis } \\
\hline No medical condition & $437(100)$ & $404(100)$ \\
\hline Confirmed at initiation or during treatment & 0 & 0 \\
\hline Unconfirmed $^{\mathrm{a}}$ at initiation of or during treatment & 0 & 0 \\
\hline \multicolumn{3}{|c|}{ Contraindicated medication taken at any time while receiving treatment with agomelatine } \\
\hline \multicolumn{3}{|l|}{ Fluvoxamine } \\
\hline No medication or confirmed not concomitant medication & $436(99.8)$ & $402(99.5)$ \\
\hline Confirmed, concomitant medication & 0 & $1(0.2)$ \\
\hline Missing & $1(0.2)$ & $1(0.2)$ \\
\hline \multicolumn{3}{|l|}{ Ciprofloxacin } \\
\hline No medication or confirmed not concomitant medication & $435(99.5)$ & $403(99.8)$ \\
\hline Confirmed, concomitant medication & $1(0.2)$ & 0 \\
\hline Missing & $1(0.2)$ & $1(0.2)$ \\
\hline \multicolumn{3}{|c|}{ Contraindication related to the ALT or AST value (result $\geq 3 \times \mathrm{ULN}$ ) } \\
\hline At least one ALT or AST result test $\geq 3 \times \mathrm{ULN}$ & 0 & 0 \\
\hline
\end{tabular}

The theoretical treatment duration was used to determine whether a condition or medication occurred at initiation or during the treatment

$A L T$ alanine transferase, $A S T$ aspartate transferase, $R M M$ risk-minimisation measure, $U L N$ upper limit of normal

${ }^{a}$ Date of diagnosis was incomplete, which means that it was not possible to verify concomitance with agomelatine exposure

\subsection{Patient Survey Results}

Of the 237 patients surveyed, $72.6 \%$ were aware of the potential liver adverse effects of agomelatine and $84 \%$ were informed about the liver-monitoring requirement. The patient's physician was the primary source of information about agomelatine risks for $76.5 \%$ of patients of GPs and $79.9 \%$ of patients of specialists (data not shown).

Overall, 182 patients (76.8\%) completing the survey indicated that they had a blood test before starting treatment with agomelatine (data not shown); $63.2 \%$ of them identified that the test was to check liver function. In addition, 194 patients $(81.9 \%)$ indicated they had a blood test $d u r i n g$ treatment with agomelatine; $78.4 \%$ of them identified that the test was to check liver function. The most frequent reason patients cited for not having a blood test performed before agomelatine treatment initiation (23 patients) or while on treatment (18 patients) was that the test was ordered but not yet performed (Electronic Supplementary Material, Table S-3).

\section{Discussion}

This study aimed to assess the effectiveness of the aRMMs for agomelatine introduced following the EMA evaluation in November 2014, in terms of adherence to the livermonitoring test requirements and compliance with relevant contraindications. Overall, adherence to the liver-monitoring recommendations did not improve after the implementation of the aRMMs, except for those required at dose escalation. However, the study showed that the contraindications and precautions of use were largely followed even before the updated prescriber guide and dissemination of the patient booklet, potentially indicating that physicians are aware of the hepatic risk related to agomelatine and, more importantly, appropriately exclude patients at high risk of hepatic injury.

Approximately $60 \%$ of patients had at least one liver test during treatment, and each of the tests required at specific timepoints during treatment was performed in approximately a third of patients still on agomelatine. The percentage of patients who had all required liver tests (at initiation and 
Table 3 Prevalence of risk factors for hepatic injury mentioned as special warning or precaution of use in the summary of product characteristics by study period: overall patients

\begin{tabular}{|c|c|c|}
\hline \multirow{2}{*}{$\begin{array}{l}\text { Risk factors for hepatic injury present at initial agomelatine pre- } \\
\text { scription and/or that occurred while taking agomelatine }\end{array}$} & \multicolumn{2}{|l|}{ Overall patients $[n(\%)]$} \\
\hline & $\begin{array}{l}\text { Before-RMM period } \\
(n=437)\end{array}$ & $\begin{array}{l}\text { After-RMM } \\
\text { period }(n=404)\end{array}$ \\
\hline \multicolumn{3}{|l|}{ Non-alcoholic fatty liver disease } \\
\hline No medical condition or confirmed outside treatment & $436(99.8)$ & $402(99.5)$ \\
\hline Confirmed at initiation or during treatment & 0 & 0 \\
\hline Unconfirmed $^{\mathrm{a}}$ at initiation of or during treatment & $1(0.2)$ & $2(0.5)$ \\
\hline \multicolumn{3}{|l|}{ Alcohol use disorder } \\
\hline No medical condition or confirmed outside treatment & $415(95.0)$ & $393(97.3)$ \\
\hline Confirmed at initiation or during treatment & $3(0.7)$ & $2(0.4)$ \\
\hline Unconfirmed $^{\mathrm{a}}$ at initiation of or during treatment & $19(4.3)$ & $9(2.2)$ \\
\hline \multicolumn{3}{|l|}{ Obesity/overweight } \\
\hline No medical condition or confirmed outside treatment & $356(81.5)$ & $331(81.9)$ \\
\hline Confirmed at initiation or during treatment & $12(2.7)$ & $15(3.7)$ \\
\hline Unconfirmed $^{\mathrm{a}}$ at initiation of or during treatment & $69(15.8)$ & $58(14.4)$ \\
\hline \multicolumn{3}{|l|}{ Diabetes mellitus (either type 1 or type 2 ) } \\
\hline No medical condition or confirmed outside treatment & $417(95.4)$ & $378(93.6)$ \\
\hline Confirmed at initiation or during treatment & $11(2.5)$ & $9(2.2)$ \\
\hline Unconfirmed $^{\mathrm{a}}$ at initiation of or during treatment & $9(2.1)$ & $17(4.2)$ \\
\hline
\end{tabular}

The theoretical treatment duration was used to determine whether a condition or medication occurred at initiation or during the treatment

$R M M$ risk-minimisation measure

a Date of diagnosis was incomplete, which means that it was not possible to verify concomitance with agomelatine exposure during treatment) was approximately $7 \%$ in both periods. The improvement in adherence to liver monitoring in patients with dose escalation suggests that prescribers are aware of the increased risk with the $50 \mathrm{mg}$ dose. The study did not collect information from physicians on the reasons for ordering a liver test or not. Nevertheless, it may be hypothesised that liver tests are more frequently ordered in the most at-risk patients or situations.

The pre-/post-RMM approach of this study was appropriate to evaluate the immediate impact of short-term programmes (e.g. 12-18 months) such as RMMs [5, 6]. Limited improvements were observed between the study periods, suggesting that the aRMMs had a low impact; however, most RMMs, including contraindications, precautions of use and liver monitoring, were already in place in November 2014.

A recent systematic review of studies evaluating the effectiveness of RMMs highlighted common challenges in designing and conducting these studies, including low response rates, limited generalisability of results and inconsistencies in reporting [7]. More specifically, the challenges of conducting studies to evaluate RMMs around potential hepatic risk are recognised in the literature, and these studies have generally found suboptimal compliance with livermonitoring requirements [8-10].
Accordingly, the results of this study must be considered in view of several limitations. Similar to other RMM evaluations [11-13], this study experienced recruitment challenges that made it difficult to draw robust conclusions [14]. A key consideration is that the study participation response rate for physicians was low (1\%). We chose to use the most comprehensive lists of prescribers available to avoid the use of lists of prescribers affiliated with the sponsor to minimise the potential for selection bias. Further, obstacles in the identification of patients and the need to obtain patient consent and access and abstract medical records may have influenced physicians' participation. Despite all efforts to involve prescribers, the number of active sites was low, particularly in Denmark and for Spanish and German GPs, potentially affecting representativeness and generalisability of the findings. Dependence on existing medical records was further impacted by shared patient management (shared care), reported for $19.7 \%$ of patients in the before-RMM and $23.8 \%$ of patients in the after-RMM period. The percentage of patients with a missing or incomplete test result date was higher in patients with shared care than in patients with same care (i.e. $51.2 \%$ in the before-RMM period and $40.6 \%$ in the after-RMM period for at least one missing date with shared care vs. $31.1 \%$ and $29.5 \%$, respectively, with same care). An additional impact of shared care was that some liver tests could have been done without the participating physicians' 
awareness or subsequent record in the patient's file. Further, although formal comparisons were not conducted, results suggest that compliance was slightly higher in each study period for specialists than for GPs, but similar changes in liver test monitoring compliance were observed when comparing the after and before periods for these two groups.

Finally, no conclusions can be drawn from the survey regarding the reasons blood tests were not performed, due to the low number of patients indicating that a blood test was not done at treatment initiation $(n=23)$ or while taking agomelatine $(n=18)$ and the diversity of answers (multiple answers were possible). However, despite its limitations, the survey indicated that nearly three-quarters of patients knew that liver problems could be potential adverse effects of agomelatine. Patients most frequently cited their physicians as a source of information about agomelatine (GP for $76.5 \%$ of GP patients and specialist for $79.9 \%$ of specialist patients), suggesting that physicians communicate with patients about agomelatine and its potential risks.

The PASS results should be considered alongside the results of two studies quantifying the risk of hospitalisation for acute liver injury with agomelatine versus other antidepressants [15]. A case-control study nested in a cohort of 3.2 million new users of antidepressants in Spain, Germany, Denmark and Sweden showed agomelatine was not associated with a higher risk of acute liver injury hospitalisation than citalopram. Similarly, a study conducted by the French Medical Agency (ANSM) in the French Health Insurance database (SNIIRAM) to evaluate the hepatotoxicity of antidepressants found no increased risk of serious liver injury associated with agomelatine relative to selective serotonin reuptake inhibitors [16]. These two studies, performed while the RMMs were in force, showed the risk of hepatotoxic reaction among agomelatine users is not higher than that for users of citalopram or other antidepressants. Additional assessments of the usefulness of the RMMs (patient booklet and prescriber's guide) are also planned. Results from these analyses are expected in the next Periodic Safety Update Report for agomelatine, anticipated in February 2021.

\section{Conclusions}

In this study to assess the effectiveness of the RMMs for agomelatine, overall adherence to liver-monitoring recommendations remained weakly influenced by the latest additional RMMs. However, 99\% of patients were treated with respect to the relevant contraindicated co-morbidities (active liver disease and cirrhosis) and concomitant contraindicated medications. Adherence to contraindications, precautions of use and blood liver testing before and during treatment as specified in the Summary of Product Characteristics is essential when agomelatine is prescribed.
Acknowledgements The authors gratefully acknowledge Manel Pladevall-Vila of RTI Health Solutions for his contributions to designing the study and developing the protocol, John Forbes of RTI Health Solutions for medical editing services, and Kate Lothman of RTI Health Solutions for assistance with developing the manuscript under the authors' direction. The authors gratefully acknowledge Kantar Health for their contributions to field operations for this study.

\section{Compliance with Ethical Standards}

Funding Financial support for this study was provided by Servier. Open access fees were paid by Servier.

Conflict of Interest This study was performed under a research contract between RTI Health Solutions and Institut de Recherches Internationales Servier and was funded by Institut de Recherches Internationales Servier. The contract between RTI Health Solutions and Institut de Recherches Internationales Servier included independent publication rights. Emmanuelle Jacquot, Estelle Collin and Nicolas Deltour are salaried employees of Institut de Recherches Internationales Servier. Amy Ladner, Anita Tormos, Lynne Hamm, Susana Perez-Gutthann, Lia Gutierrez and Costel Chirila are salaried employees of RTI Health Solutions.

Ethical Approval All appropriate approvals from national and local ethics committees and other competent authorities were obtained for this study.

Informed Consent For the medical record abstraction (MRA) phase of the study, patients whose medical records were abstracted provided written informed consent (except in France, where only the person not objecting to the processing of his or her personal data for the purposes of the MRA was required). For the survey phase of the study, all participating patients provided written informed consent.

Open Access This article is distributed under the terms of the Creative Commons Attribution-NonCommercial 4.0 International License (http://creativecommons.org/licenses/by-nc/4.0/), which permits any noncommercial use, distribution, and reproduction in any medium, provided you give appropriate credit to the original author(s) and the source, provide a link to the Creative Commons license, and indicate if changes were made.

\section{References}

1. Servier. Integrated analyses of safety. (2015) (Data on file).

2. European Medicines Agency. Valdoxan: scientific conclusions and grounds recommending the variation to the terms of the marketing authorisation. 2014. http://www.ema.europa.eu/docs/en_GB/ document_library/EPAR_-_Scientific_Conclusion/human/00091 5/WC500179069.pdf. Accessed 09 Jun 2015.

3. Newcombe RG. Interval estimation for the difference between independent proportions: comparison of eleven methods. Stat Med. 1998;17(8):873-90.

4. Fisher LD, van Belle G. Categorical data: contingency tables. In: Fisher LD, van Belle G, editors. Biostatistics: a methodology for the health sciences. New York: Wiley Interscience; 1993. p. 246-303.

5. Carroll R, Ramagopalan SV, Cid-Ruzafa J, Lambrelli D, McDonald L. An analysis of characteristics of post-authorisation 
studies registered on the ENCePP EU PAS Register. F1000Res. 2017;6:1447.

6. de Belvis AG, Barbara A, Giubbini G, Traglia S, Angioletti C, Ianiro $\mathrm{G}$, et al. Impact evaluation of a critical pathway for patients with Clostridium difficile infection: a pre-post analysis in a Third Level Referral Center. Int J Infect Dis. 2019;80:105-10.

7. Artime E, Qizilbash N, Garrido-Estepa M, Vora P, SorianoGabarro M, Asiimwe A, et al. Are risk minimization measures for approved drugs in Europe effective? A systematic review. Expert Opin Drug Saf. 2019;18(5):443-54.

8. Cluxton RJ Jr, Li Z, Heaton PC, Weiss SR, Zuckerman IH, Moomaw CJ, et al. Impact of regulatory labeling for troglitazone and rosiglitazone on hepatic enzyme monitoring compliance: findings from the state of Ohio medicaid program. Pharmacoepidemiol Drug Saf. 2005;14(1):1-9.

9. Shantakumar S, Nordstrom BL, Hall SA, Djousse L, van HerkSukel MPP, Fraeman KH, et al. Prescriber compliance with liver monitoring guidelines for pazopanib in the postapproval setting: results from a distributed research network. J Patient Saf. 2019;15(1):55-60.

10. US Food and Drug Administration. Risk assessment and risk mitigation review(s): application number 022406Orig1s000 2009. https://www.accessdata.fda.gov/drugsatfda_docs/nda/2011/02240 6Orig1s000RiskR.pdf. Accessed 07 Jun 2019.

11. Davis KH, Asiimwe A, Zografos LJ, McSorley DJ, Andrews EB. Evaluation of risk-minimization activities for cyproterone acetate $2 \mathrm{mg} / \mathrm{ethinylestradiol} 35 \mathrm{mcg}$ : a cross-sectional physician survey. Pharm Med. 2017;31(5):339-51.
12. Madison T, Huang K, Huot-Marchand P, Wilner KD, Mo K. Effectiveness of the crizotinib therapeutic management guide in communicating risks, and recommended actions to minimize risks, among physicians prescribing crizotinib in Europe. Pharm Med. 2018;32(5):343-52.

13. Landsberg W, Al-Dakkak I, Coppin-Renz A, Geis U, PetersStrickland T, van Heumen E, et al. Effectiveness evaluation of additional risk minimization measures for adolescent use of aripiprazole in the European Union: results from a post-authorization safety study. Drug Saf. 2018;41(8):797-806.

14. Banerjee AK, Zomerdijk IM, Wooder S, Ingate S, Mayall SJ. Postapproval evaluation of effectiveness of risk minimisation: methods, challenges and interpretation. Drug Saf. 2014;37(1):33-42.

15. Pladevall M, Hallas J, Schink T, Reutfors J, Morros R, PobladorPlou B, et al. Agomelatine and other antidepressants and the Risk of acute liver injury (ALI), a PASS in Four European countries. Pharmacoepidemiol Drug Saf. 2018;27(S2):19-20.

16. de Billioti Gage S, Collin C, Le-Tri T, Pariente A, Begaud B, Verdoux H, et al. Antidepressants and hepatotoxicity: a cohort study among 5 million individuals registered in the French National Health Insurance Database. CNS Drugs. 2018;32(7):673-84. 